\title{
Evidence-based medicine; climbing a mountain for a better decision-making
}

\author{
Mona O. Mohsen, Ahmed M. Malki and Hassan Abel Aziz* \\ Biomedical Science Program, Department of Health Sciences, College of Art and Sciences, Qatar University, Doha, Qatar
}

\begin{abstract}
Evidence-Based Medicine is a relatively new term used in medical sittings and Health Information Technology (HIT). It is a form of medicine that integrates practitioners' expertise with the best available practical evidences to improve better patient care. Evidence-Based Medicine has increasingly been used and incorporated into daily medical practices to overcome the shortcomings in the conventional standard care. The purpose of this literature review is to highlight the importance of Evidence-Based Medicine and how it can act as a crucial tool in decision-making to empower the quality of medical services for better patient outcomes.
\end{abstract}

\section{Introduction}

Clinicians face a myriad of medical scenarios every day. In one examination room, a clinician has just seen a 52-year-old smoker female who has been recently diagnosed with advanced squamouscell carcinoma in the oral cavity [1]. The clinician decided that the tumor was amenable to surgery; however, a visiting medical student recalled from a recent lecture that chemotherapy was effective in this case and could significantly improve the survival rate for this patient. The clinician challenged this information whether using neo-adjuvant chemotherapy would be beneficial for locally advanced squamous-cell carcinoma or if it would enhance the survival rate. The clinician was in an urgent need for a credible reference to avoid a potentially fetal medical error [1].

Evidence-Based Medicine (EBM) is an inter-disciplinary approach to medicine that emphasizes the utilization of evidences and conducted researches to optimize the decision-making and improve patient care outcomes [2]. EBM integrates practitioners' expertise with the best available evidences and resources and it is based on the strongest types of researches such as systemic reviews, meta-analysis and randomized controlled trials that can yield strong and reliable recommendations [2].

In the Seventeenth century the Renaissance Era of EBM has roughly began when textbooks and personal journals become more prominent. The period between 1900 and 1970s was called the "Translational Era" of $E B M$ as sharing knowledge from textbooks and peer-reviewed journals became much easier. In the 1970s, the Modern Era of EBM began where technology took a bigger role in the development and advancement of EBM, using computers, database software, and Index Medicus [3]. In more recent years, the term was defined by investigators from McMaster's University during the 1990s as " $a$ systemic approach to analyze published research as the basis of clinical decision making" [4]. Later, Sacket et al. formally defined the term in 1996 as "the conscientious and judicious use of current best evidence from clinical care research in the management of individual patients" [4]. The Center for Evidence- Based Medicine (CEBM) described the term as "the conscientious, explicit, and judicious use of current best evidence in making decisions about the care of individual patients,
The practice of evidence based medicine means integrating individual clinical expertise with the best available external clinical evidence from systematic research" [5].

\section{Evidence based medicine process}

A clinician should follow these typical steps to reach a decision (Table 1) [6]:

Filtered resources are usually used to appraise the quality of search and to allow practice recommendations. For example, The Cochrane Database, The Database of Abstracts of Reviews of Effects (DARE), PubMed and Ovid MEDLINE are good sources of systemic reviews and meta-analysis studies. DynaMed, National Guideline Clearinghouse are used mainly for Critical-Appraised Topics while, The ACP Journal Club, Evidence Updates, Dartmouth EBM Database and EvidenceBased Journal Series are Critically-Appraised Individual Articles.

\section{Evidence-based medicine important tool in decision- making}

Robert E. Hoyt and William R. Hersh stated that, "Learning EBM is like climbing a mountain to gain a better view. One might not make it to the top and find the perfect answer but individuals will undoubtedly have a better vantage point than those who choose to stay at sea level" [7]. The European Surgical Research journal has published in 2014 a survey-based comparison between UK and Germany to analyze the attitude of their surgeons towards $E B M$, all participants considered $E B M$ as a vital tool for everyday decision-making, as well as for patients and overall health system [8].

There are several reasons to incorporate EBM in today's practice, for

Correspondence to: Hassan Abdel Aziz, Biomedical Science Program, Department of Health Sciences, College of Art and Sciences, Qatar University, Doha, Qatar, Tel: 0974-4403-4783; E-mail: hassan.aziz@qu.edu.qa

Key words: EBM: evidence-based medicine, CEBM: center for evidence-based medicine, HIT: health information technology, CPGs: clinical practice guidelines

Received: March 25, 2015; Accepted: April 12, 2015; Published: April 15, 2015 
Table 1. The steps to process an evidence-based decision.

\begin{tabular}{|c|c|}
\hline The Patient & Start with a clinical problem arises from the case \\
\hline The Question & $\begin{array}{l}\text { Construct and formulate the clinical question according to PICO method, PICO is a well-built question consists of four main parts (P: } \\
\text { Population or Participants - I: Intervention or Indicator - C: Comparator or Control and O: Outcome) developed by the national Library of } \\
\text { Medicine } \\
\text {. What is the case? } \\
\text { - What intervention, treatment, diagnostic tests, etc.? } \\
\text { What type of comparison, treatment A versus B, placebo? }\end{array}$ \\
\hline The Resource & 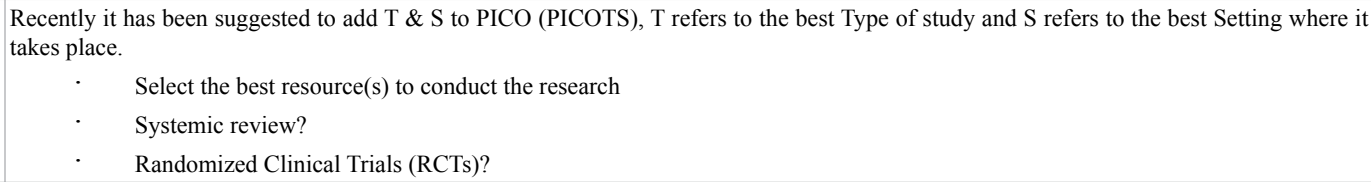 \\
\hline The Evaluation & Critically appraise for internal \& external validity, significance, applicability \& impact of intervention. \\
\hline The Patient & Integrate the EBM with the clinical expertise; apply to practice, patient's values and circumstances. \\
\hline Self-Evaluation & $\begin{array}{l}\text { Evaluate your performance and assess the outcome } \\
\begin{array}{cl}\text {. } & \text { Right question? } \\
\text {. } & \text { Better outcome? } \\
\text {. } & \text { Clinical sense? }\end{array}\end{array}$ \\
\hline
\end{tabular}

instance the medically and educationally used methods are not always working, the slow process of translational researches, also some of the daily "standard of care" practice are challenged and may be wrong. EBM is dynamic as the knowledge gained from clinical trials can be applied directly to patient's care, EBM provides consistency in patient's care and treatment that assume the optimal clinical outcome and improve the quality of a patient's life [9]. EBM looks for the strength behind medical interventions and clinical trials in terms of benefits and risks, and thus it is an important tool in decision making at the individual level and for the population at large. Therefore, it is considered a crucial and emerging medical care tool to ensure better outcomes for patients. Measuring clinician practice and the patient's outcome can be a challenge as patients vary in their degree of illness and complexity such as the individual response to treatment and medication, $E B M$ will help clinicians to provide care that is more rational for the patients [10]. $E B M$ is considered the new paradigm for medical care and for reaching informed clinical decisions; it emphasizes the importance of large clinical trial results and setting individual treatment strategies. Physician usually make their decisions based on their experience and practice years, these ways may be time efficient but would not provide best care decision when it comes to the patient. In EBM, physicians seeking additional information pose questions that guide research for ideal literature, during a normal single day for physician, he/she can generate as many as eight clinical questions, finding the correct answer can alter the physician way of management [10].

A recent study was conducted to identify best practices and evidence based interventions for patients with autism disorder, a preliminary search for $E B M$ yielded about thirty thousand articles. Further screening resulted in 456 studies that meet the inclusion and methodological criteria. This was also followed by focused research in intervention practices lead about 27 articles only; the author was successful in describing six new evidence based practices that assess autism patients, as well as new implementation for current and future practices [11].

In another example, a group of plastic surgeons at Georgetown University published a recent study in 2013 based on EBM. The aim of the study was to help participants identifying the most common facial skin malignancies in order to be familiar with the best-recommended surgical treatment and update them about the recurrence rate, prognosis and mortality [12].
$E B M$ can be further linked to medical care as illustrated in Figure 1. Good medical performance is based on quality and quality depends on the best available $E B M$. $E B M$ focuses more on doing the right things while quality of care and improvement focuses mainly on doing things right to make sure that actions are done in the most reliable and efficient way [13]. Combining both will result in "doing the right things right" [13]. EBM is an engine that measures the health care practices and identify the gaps found in health care organizations between clinical evidence and health practices. It plays a major role in capturing knowledge about quality care management at certain organization and compares the actual observed patient care with care indicated by the guidelines of specific research evidence based [14]. These findings can be used by the organizations to identify diseases and care opportunities and reach conclusions about the medical condition at the individual and at the population in general. The findings can be extended to identify physicians and practitioners with high performance and the areas that need to improve compliance. $E B M$ can help in identifying tests and treatments that are harmful to patients and/or not necessary. Moreover, it can identify patients with poor control of the disease and can reduce potentially harmful treatments. EBM addresses the criteria that organizations use to apply quality measurement built around national standards in order to provide robust guidelines to measure compliance (Figure 1) [6].

William C. Livingood at the University of Florida College of Medicine-Jacksonville (Center for Health Equity \& Quality Research) published in February 2015 an evaluation on a Georgia Childhood Asthma Management Program, a Healthcare Georgia Foundationsupported initiative for multiple diverse programs and settings [15]. The author used evidence-based medicine, quantitative and qualitative analysis and data collection. The aim of this study was to assess the programs in building community, especially where it was starting, the changes on the level-systems and the progress towards more evidencebased community. The initial results of this study revealed that there were minimum efforts towards assessing the health outcome and the changes in the system; evidence-based medicine was poorly linked with the patients' health outcome. He introduced a developmental technique to develop logic models able to link practices to outcomes that could be used with any community sitting [16].

The development of standard and applicable guidelines is a main 


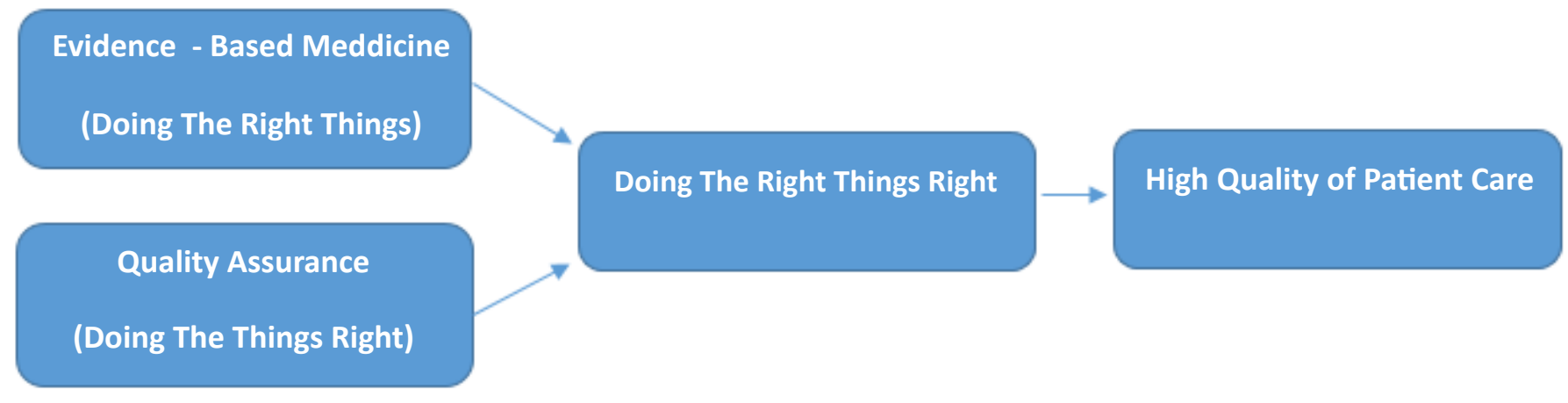

Figure 1. Combining EBM with Quality would result in doing the right things right and improve overall quality of the health system.

benefit of $E B M$ especially when discussing the prescribing criteria. The guidelines make it more efficient in order to review treatment strategies, promote cost effective decisions and study the value of the guidelines to manage issues and to support policies [17]. The Institute of Medicine (IOM) has defined clinical Practice Guidelines (CPGs) in 1990 as "systematically developed statements to assist practitioner and patient decisions about health care for specific clinical circumstances". CPGs are logical steps of transforming the evidence-based medicine into recommendations, used by most organizations to improve the quality of care, reduce costs, while maintaining patient safety. The guidelines are supported by the HIT allowing for fast and easy search for evidences and facilitates its linkage to Electronic Health Records (EHRs). A recent study has shown that CPGs that address chronic diseases can work effectively. A 50\% decrease in both cardiovascular and microvascular complications were observed in a study of patients with diabetes after implementing CPGs and intensive treatment of several risk factors [17]. In 2006, Circulation Journal published a study entitled "Evidence-Based Medicine: Making the Grade" by "The Center for Cardiovascular Science and Medicine in the University of North Carolina" [18]. The article was initiated with a catching quote "Rarely has cardiovascular medicine faced greater challenges. Rarely have our patients expected more of us. However, never have we had such tools to make people well.... It is time to make good on that promise". The author discussed two studies that provide important information on the use of aspirin, statins, ACE-Inhibitors and b-Blockers among patients suffering from coronary artery diseases and hypertension, the "The Institute of Medicine" had observed major deficiencies in health care provided for those patients and had emphasized the importance of implementing and utilizing evidence-based therapies. Both studies revealed vital information about the use of these therapies and highlighted the importance on focusing on new strategies to ensure patients' adherence to recommended therapies [18]. Results from both studies were carefully considered by several American organizations such as the American Heart Association, and National Quality Improvement and American College of Cardiology where guidelines have been implemented to improve health outcomes of cardiovascular patients [18].

\section{Challenges}

Thomas Huxley stated, "The great tragedy of Science is the slaying of a beautiful hypothesis by an ugly fact" [7]. As the case in all new technologies there are always challenges and obstacles. EBM is at the interface of several challenges. One challenge is the gigantic amount of available data or "big data", creating datasets for big data is a complex process, costs a lot of money and requires continuous maintenance and security. An average individual physician is faced annually with more than thirty thousand published biomedical journals and more than seventeen thousand medical books, making it extremely challenging for clinicians with time and ability to cope, even in a specialist area [19]. In 1992, more than 6000 articles in adult internal medicine were published; a physician has to read daily about 20 related articles to keep with the updates. This number has increased dramatically in our days, as more than 700,000 articles are added to Medline yearly and the physician's desk reference (PDR) is over 3000 pages long. Sacket et al. suggested that the average practicing clinician spend these days less than one hour weekly reading evidence based information, even if these physicians are familiar with $E B M$ articles and how to critically grade and assess them, they may not be able to answer many of their patients questions [7]. Therefore, busy physicians and clinicians need to learn new technologies that would make practicing $E B M$ more feasible [19]. Using EBM requires new skills on the part of the clinicians such as efficient and frequent literature review searches and to critically use and establish rules to evaluate the clinical literatures. Furthermore, translating the evidence research based medicine into a routine practice for everyday use is a major challenge, due to the gap between the routine patient care and the practice suggested by the EBM [17].

Several approaches by a number of organizations are underway to address the challenges of implementing EBM in clinical and academic sittings. For example, Boston University School of Medicine has published in 2015 a custom-made tool called Finding Information Framework (FIF) to help students and learners utilizing EBM resources to reach best clinical decisions [12]. FIF is a conceptual algorithmic tool that helps students to categorize questions and link them directly to best available resources. It categorizes the questions to background and foreground questions upon the student choice. The tools utilizes textbooks and PubMed for basic science questions, UpToDate for clinical questions and DynaMed for foreground inquiries and point of care questions. FIF is available on mobile applications [12].

\section{Conclusion}

$E B M$ is a buzz term in the medical field and it is an increasingly important tool to cope with the information overload and evidences in order to provide the best patient care. Several recent researches and studies have shown the significance and importance of $E B M$ in decision-making and in improving the quality of healthcare. $E B M$ can provide a vital guidance to clinical situations and cases. 


\section{References}

1. Hunt DL, Haynes RB, Browman GP (1998) Review on evidence-based cancer medicine Searching the medical literature for the best evidence to solve clinical questions. Annals of Oncology 9: 377383.

2. Evidence Based Medicine (2014) University of South Dakota.

3. Clariadge JA, Fabian TC (2005) History and Development of Evidence-Based Medicine. World J Surg 29: 547-553. [Crossref]

4. Evidence-Based Medicine Working Group (1992) Evidence-based medicine. A new approach to teaching the practice of medicine. JAMA 268: 2420-2425. [Crossref]

5. CEBM Center for Evidence Based Medicine (2014).

6. Glasziou P, Orgrinc G, Goodman S (1996) Evidence Based Medicine. BMJ 312: 71-72.

7. Hoyt E, Hersh R. Evidence Based Medicine and Clinical Practice Guidelines 14: 324344.

8. Schnitzbauer A, Proneth A, Pengel L, Ansorg J, Anthuber M, et al., (2015) EvidenceBased Medicine in Daily Surgical Decision Making: A Survey-Based Comparison between the UK and Germany. Eur Surg Res 54: 14-23. [Crossref]

9. Using Evidence Based Medicine to Improve Health Care 2008.

10. Evidence Based Medicine (2014) University of South Dekota.
11. Evidence-Based practice for Children (2015) Youth and Young Adults with Autism Spectrum Disorder: A Comprehensive Review. J Autism and Development Disorder.

12. Annie L, Theresa A, David B. Novel Mobile Application to Promote Evidence-Based Medicine Decision-Making in Medical Education.

13. Glasziou P, Orgrinc G, Goodman S (2011) Can Evidence-Based Medicine and Clinical Quality Improvement learn from each other? BMJ Qual Saf: i13-i17.

14. Gustave L, Janet W (2014) Evidence Based Medicine, Icahn School of Medicine at Mount Sinai.

15. Woodhouse L, Livingood W, Toal R, Keene D, Hines H, et al. (2015) Evaluation of Diverse Community Asthma Interventions: Balancing Health Outcomes with Developing Community Capacity for Evidence-Based Program Measurement. Popul Health Manag. [Crossref]

16. Iorio, Matthew L, Ter Louw, Ryan P, Kauffman, et al. (2013) Evidence-Based Medicine: Facial Skin Malignancy. Plast Reconstr Surg 132: 1631-1643. [Crossref]

17. Matthew J, McQueen (2001) Overview of Evidence Based Medicine: Challenges for Evidence Based Labortory Medicine. Clin Chem. 47: 1536-1546. [Crossref]

18. Sidney C, Smith J (2006) Evidence-Based Medicine: Making the Grade. Circulation 113: 178-179.

19. Eric W (2014) Challenges to using evidence-Based Medicine in Daily Clinical Practice.

Copyright: $@ 2015$ Mohsen MO. This is an open-access article distributed under the terms of the Creative Commons Attribution License, which permits unrestricted use, distribution, and reproduction in any medium, provided the original author and source are credited. 